NOTE

\title{
Solar ultraviolet radiation response of EBT2 Gafchromic, radiochromic film
}

\author{
Ethan T Butson $^{1,3}$, Peter K N Yu ${ }^{2}$ and Martin J Butson ${ }^{2}$ \\ ${ }^{1}$ The Illawarra Grammar School (TIGS), Western Ave, West Wollongong, NSW, Australia \\ 2 Department of Physics and Materials Science, City University of Hong Kong, Kowloon Tong, \\ Hong Kong \\ E-mail: diamonds.for.you@hotmail.com
}

Received 25 February 2013, in final form 8 September 2013

Published 11 October 2013

Online at stacks.iop.org/PMB/58/N287

\begin{abstract}
Measurement of solar ultraviolet (UV) radiation is an important aspect of dosimetry for the improved knowledge of UV exposure and its associated health related issues. EBT2 Gafchromic film has been designed by its manufacturers as an improved tool for ionizing radiation dosimetry. The film is stated as exhibiting a significant reduction in UV response. However, results have shown that when exposed to UV from the 'bottom side' i.e. from the thick laminate side, the film exhibits a sensitivity to solar UV radiation which is both measurable and accurate for UV dosimetry. Films were irradiated in this position to known solar UV exposures and results are quantified showing a reproducibility of measurement to within $\pm 7 \%$ (1 SD) when compared to calibrated UV meters. With an exposure of $20 \mathrm{~J} \mathrm{~cm}^{-2}$ broad spectrum solar $\mathrm{UV}$, the films net OD change was found to be $0.248 \mathrm{OD} \pm 0.021 \mathrm{OD}$ when analysing the results using the red channel region of an Epson V700 desktop scanner. This was compared to $0.0294 \mathrm{OD} \pm 0.0053 \mathrm{OD}$ change with exposure to the same UV exposure from the top side. This means that solar UV dosimetry can be performed using EBT2 Gafchromic film utilizing the underside of the film for dosimetry. The main advantages of this film type for measurement of UV exposure is the visible colour change and thus easy analysis using a desktop scanner as well as its uniformity in response and its robust physical strength for use in outside exposure situations.
\end{abstract}

\section{Introduction}

Solar ultraviolet (UV) radiation is becoming increasingly dangerous to people on earth as their magnitude increases caused by effects such as the ozone layer depletion (Allen et al 1998).

3 Author to whom any correspondence should be addressed. 
The wavelength of UV is in-between the visible light spectrum and the X-ray spectrum (Diffey 1982). UV is emitted from our sun in three different forms, UV-A, UV-B and UV-C. These three forms of UV make up the UV spectrum. Historically UV-A is defined as the wavelength that 'ages' the skin. UV-B was defined as the wavelength that 'burns' the skin and UV-C defined as the 'cancerous' wavelength. The wavelength ranges for UV-A, UV-B and UV-C are $A=400-315 \mathrm{~nm}, B=315-280 \mathrm{~nm}$ and $C=280-100 \mathrm{~nm}$ ) (ISO 2007). Today, it is known that all forms of UV radiation in excessive amounts are detrimental to humans.

Some forms of UV radiation dosimeters have included UV sensitive films. Schouten et al (2009) researched and investigated the ability to measure UV-A radiation with a film type detector called polyphenylene oxide film. Polyphenylene oxide is a high temperature thermoplastic material which undergoes absorpbance spectral changes when exposed to UV radiation. Parisi and Kimlin (2004) and Schouten et al (2007) used a film type called polysulfone (high temperature thermoplastic utilizing a sulfate, $\mathrm{SO}_{2}$, subunit) film to measure UV dose in underwater environments and in land environments. These types of film detectors have been used to assess the UV exposure to humans but they also can have disadvantages which are mentioned below. These films can only be read out for UV dose assessment using an UV radiation light source and special, photo spectrometer equipment. This means that it does not actually change colour in the visible region but has to be scanned in the UV spectrum to find out the exposure level. This can only be done using specialized photo spectrometer equipment, which is not readily available to the local community.

In recent years, ISP, Gafchromic film EBT was utilized for solar UV radiation measurement (Butson et al 2010) and found to be a reproducible and adequate measure for broad band UV radiation. Since this work was performed, the manufacturing company International speciality products have changed the design of the film completely and formed a new product now named Gafchromic EBT2.

Several changes have occurred including different over laminate materials and the inclusion of a yellow dye within the active layer. The active radiochromic compound however remains the same. The manufacturers have quoted that the film is now much less sensitive to UV radiation to allow use under normal fluorescent light sources inside.

This work however has discovered by using the underside of the film as the 'active' measurement tool, solar UV radiation is still measureable in a quantitative and accurate way. This work investigates the solar UV measurement ability of Gafchromic EBT2 film.

\section{Materials and methods}

Experiments were performed using ISP Gafchromic EBT2 (Ashland Inc., Covington, KY, USA, 2012) film (Lot no A04181102A) (expiry date April 2013) to assess the response to UV radiation of the film and provide a quantitative relationship per unit exposure. The films physical attributes are shown in figure 1 which has been adapted from ISP's white paper on Gafchromic EBT2 (ISP Corp 2009). Of note for this work is the construction of EBT2 film whereby different thicknesses and materials are present in the films construction both above and below the sensitive layer.

It is acknowledged that Gafchromic films are known to be sensitive to x-ray radiation (Butson et al 1998a, 1998b, Cheung et al 1998, 2006) however the contribution from solar $\mathrm{x}$-ray radiation or background radiation was considered negligible for these experiments.

To assess the response of the film to UV radiation, various tests were performed. Firstly the films were exposed to solar radiation in Mangerton, NSW Lat $34^{\circ} 26^{\prime} \mathrm{S}$, Long $150^{\circ}$ $52^{\prime} \mathrm{E}$ from 10 am till $2 \mathrm{pm}$ under different sky conditions. Namely, full sun and cloudy conditions. The films were irradiated for time lengths between $5 \mathrm{~min}$ and approximately 


\begin{tabular}{|c|}
\hline Polyester over laminate (50 microns thickness) \\
\hline Adhesive (25 microns thickness) \\
\hline Topcoat (5 microns thickness) \\
\hline Active layer (30 microns thickness) \\
Polyester substrate (175 microns thickness) \\
\hline
\end{tabular}

Figure 1. Schematic of Gafchromic EBT2 film (adapted from ISP EBT2 white paper).

$4 \mathrm{~h}$ depending on the irradiance level and the exposure required. The aim was to assess variations in film response caused by these differing conditions. The film pieces were cut into $3 \mathrm{~cm} \times 3 \mathrm{~cm}$ squares for experimental procedures. Experiments were performed on a standard white matt backing material to eliminate variations in response caused by differences in UV backscattering conditions. To calibrate the UV exposure, an Optix Inc, UVTEX A+B meter (calibrated May 2011) was used to measure irradiance (power per unit area) and exposure (Irradiance integrated over time) in a position next to the EBT2 Gafchromic films. The UVtex meter measures both UV-A and UV-B radiation in the wavelength range from approximately 280 to $380 \mathrm{~nm}$ peaking in response at $350 \mathrm{~nm}$. Manufacturer's specifications state that the meter can measure UV irradiance to values of $\pm 0.1 \mathrm{~mW} \mathrm{~m}^{-2}$ with an accuracy of $\pm 5 \%$.

After the films were irradiated to known UV exposures, they were kept in light tight containers to eliminate any further UV exposure (Butson et al 1998). The films were then analysed $24 \mathrm{~h}$ after the end of the exposure experiment using reflectance scanning on an Epson V700 desktop scanner. The films were analysed using Image J software on a PC workstation. The Epson Perfection V700 photo scanner was used with a scanning resolution of 150 pixels per inch in reflection mode using a white matt backing material to improve scanner uniformity (Butson et al 2011). A minimum warm up time of $30 \mathrm{~min}$ is used with this scanner. The films are scanned within the centre $10 \mathrm{~cm} \times 20 \mathrm{~cm}$ of the scanner area, again to improve scanner uniformity. The images produced were 48 bit RGB colour images. An area of $2 \mathrm{~cm} \times 2 \mathrm{~cm}$ at the centre of the $3 \mathrm{~cm} \times 3 \mathrm{~cm}$ film pieces was used to analyse the pixel values of the film. This was performed to minimize effects of variations in optical density measurement caused by film piece edges. No filters or correction functions were applied to raw pixel value results. These images were analysed using the red component. Net reflective optical density (ROD) for all films were calculated to evaluate the colouration change due to UV exposure. Net ROD is defined as

$$
\text { Net ROD }=\log \left(P_{u} / P_{t}\right)
$$

where $P_{u}$ is the pixel value of the reflected intensity from an unexposed film and $P_{t}$ is the pixel value of the reflected intensity from an exposed film. Ohuchi (2007) produced a similar definition for reflected optical density. The experiments were repeated five times on different days. Curves were produced to establish the response of EBT2 Gafchromic film to UV radiation. Uncertainties in all results were calculated as the standard deviation of the measurements repeated over five experimental sets of data including both inter and intra film uncertainties. That is, a combination of errors associated with variations measured within the $2 \mathrm{~cm} \times 2 \mathrm{~cm}$ film piece scan associated with film and scanner variations as well as variations 


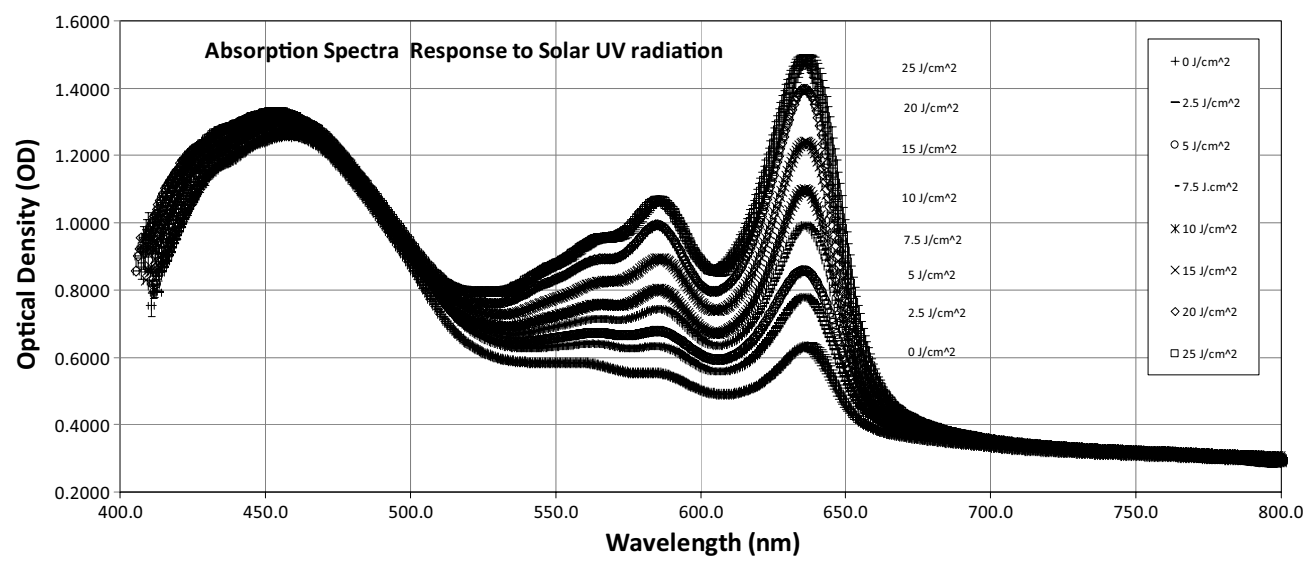

Figure 2. Absorption spectra response of EBT2 Gafchromic film to solar UV radiation.

in measured response for the five experimental procedure repeats at each measurement point. These are shown on all figures in the $y$ direction. The $x$-axis errors in figures 3-5 show the uncertainty in measured UV exposure associated with the calibrated UVTEX A+B UV meter.

The absorption spectra of the EBT2 film was analysed using an Advantes, Avaspec 2048 photo spectrometer. It was capable of measuring absorption spectra from 350 to $1100 \mathrm{~nm}$. Analysis was performed in the visible/infrared range from 400 to $800 \mathrm{~nm}$. The precision of this instrument was within $3 \mathrm{~nm}$ for wavelength analysis. The films were placed within the photo spectrometer and analysis performed using a wavelength variation of $0.5 \mathrm{~nm}$. Various films were measured with different exposure levels ranging from $0 \mathrm{~J} \mathrm{~cm}^{-2}$ up to $100 \mathrm{~J} \mathrm{~cm}^{-2}$. Results were analysed using Microsoft Excel to calculate their net ROD absorption spectra.

\section{Results and discussion}

Figure 2 shows the absorption spectra response of EBT2 Gafchromic film when exposed to solar UV radiation during summer time on a clear day. Results are given for films exposed up to $25 \mathrm{~J} \mathrm{~cm}^{-2}$, when the film is exposed with the thick laminate upwards. This fact will be discussed later. As can be seen there is a change in measured absorption spectra with increasing UV exposure with the film changing colour from a light green to dark green colour upon exposure. Variations occur at most wavelengths with the results showing the peak in absorption caused by the yellow dye at wavelengths below approximately $525 \mathrm{~nm}$. Two absorption peaks at 585 and $636 \mathrm{~nm}$ are observed, similar to the peaks caused by x-ray irradiation colouration from medical linear accelerators (Butson et al 2009). These results show that the change in colour with UV exposure are measureable and at values which would be defined as useable for UV exposure measurement. Depending on the wavelength of analysis, various sensitivities could be established for dosimetry. Figure 3 shows an example of the exposure response curves produced when analysis is performed with band pass wavelengths of $30 \mathrm{~nm}$ with centres at 535, 585 and $635 \mathrm{~nm}$. Results show that different sensitivities can be produced depending on how analysis is performed, with wavelengths closer to the absorption peaks producing higher sensitivities. This could provide an analysis method whereby the user can predefine the level of sensitivity required for a certain experiment through analysis at the specific wavelength. As can be seen, with analysis at wavelengths of 620-650 $\mathrm{nm}$ a higher sensitivity is recorded however, the film begins to saturate in response around $60 \mathrm{~J} \mathrm{~cm}^{-2}$. If the user required a larger 


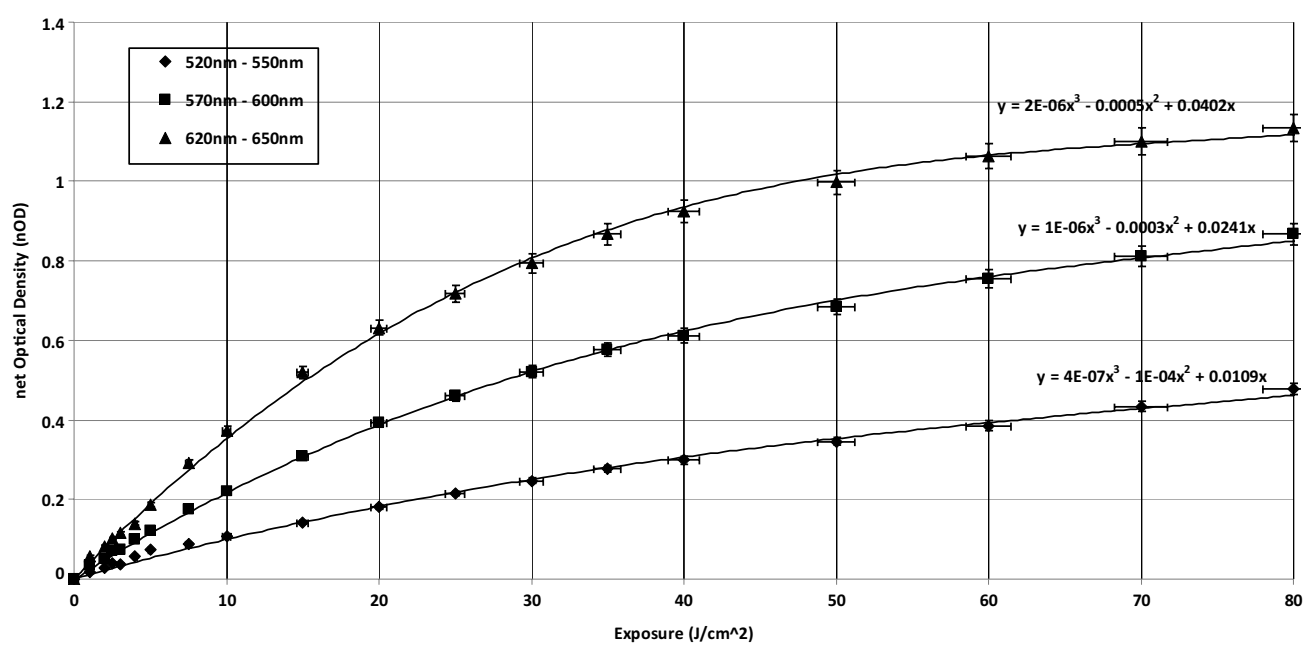

Figure 3. Sensitivity response of the film to various 'band passes' of analysis showing the ability to vary the response of the film to UV exposure.

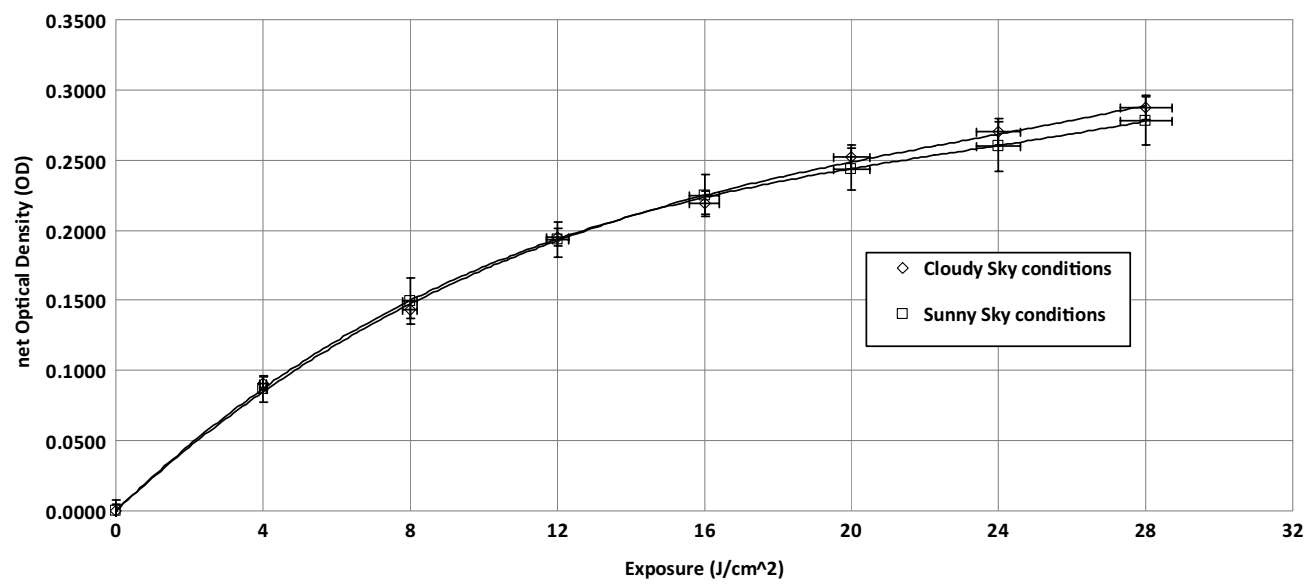

Figure 4. Response of EBT2 film to solar UV in different sky conditions such as sunny and cloudy skies.

measurement range, they would be more suited to perform analysis in say the $520-550 \mathrm{~nm}$ wavelength range where results are still viable up to $80 \mathrm{~J} \mathrm{~cm}^{-2}$ albeit at a lower sensitivity.

When analysis for UV exposure dosimetry is performed using the Epson v700 desktop scanner and the red channel, figure 4 is produced. It compares the exposure response of EBT2 film when exposed during sunny, summer time to results during cloudy, summer time conditions. These results show that at larger exposure levels, small but measureable differences were seen between cloudy and sunny results. This is most likely caused by variations in scattering angles of UV-A and UV-B in these differing sky conditions. As such, it is recommended that the calibration curve most closely suited to the sky conditions of the experiment be used. If a longer time period of evaluation is used where sky conditions change, it is recommended to use an average of the two calibration curves produced. As can be seen the response is nonlinear however is matched well by a third order polynomial fit. From these experiments, there have been two main sources of error analysed and calculated. That 


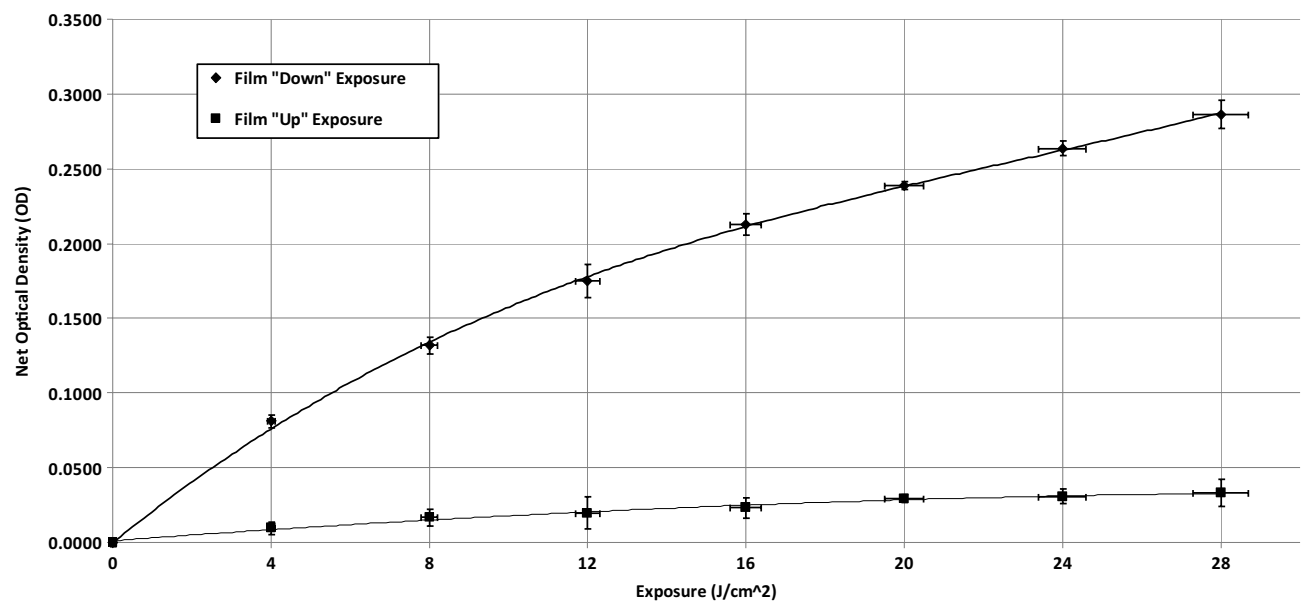

Figure 5. Comparison of EBT2 film response to UV radiation by irradiance from the topside 'thin laminate' or the bottom side 'thick laminate'.

being the uncertainty from multiple experiments (inter-film uncertainty) as well as the error in measurement caused by the analysis process itself (intra-film uncertainty) where variations in the measured pixel values occur over the scanned area of each film piece. To effectively calculate the total error, the combined error method was used such that the total error is given by the square root of the sum of the squares of each error component. Utilizing this process, an estimated maximum error in measurement was found to be $\pm 7 \%$ (1SD) and errors are shown on the figures.

One aspect of UV dosimetry which needs to be addressed is the measurement of UV radiation and the dependence of which side of the film is directly exposed to the UV source. As stated earlier, the manufacturers, in an attempt to reduce the UV response of the film when used for medical x-ray dosimetry, added a yellow dye and UV inhibitors to the films construction. This layer effectively reduces the UV response of the film. This is shown in figure 5 which is a comparison of the UV response when the films is exposed directly to UV solar radiation from the underside (thick laminate layer up) and the top side (thin laminate layer up) of the EBT2 film. As can be seen a significant difference in response is seen with the film producing a marked decrease in response with the top side placed up for exposure. For example, at an exposure level of $12 \mathrm{~J} \mathrm{~cm}^{-2}$, the net optical density change was 0.175 and 0.0198 for 'down' and 'up' exposures respectively, an approximate tenfold difference. The difference is approximately tenfold. This of course is the response that the manufacturers aimed for so that in normal medical x-ray dosimetry, the film would have a reduced response to UV light sources like fluorescent lights. This however, has not affected the films ability to accurately measure UV radiation utilizing the underside of the film.

It is acknowledged that detectors produce differing response characteristics and sensitivities with respect to UV wavelengths. That is, each detector can have differing sensitivity to say UV-A and UV-B wavelength exposures. The UVTEX A+B meter is stated by manufacturers to measure both UV-A and UV-B wavelengths (280 to $380 \mathrm{~nm}$ ) with a peak wavelength at $350 \mathrm{~nm}$. We have not studied wavelength spectral response of EBT2 film and have made direct comparisons to exposure compared to the UVTEX A+B meter. Evaluating the spectral response of EBT2 will be the next step in our investigations.

Gafchromic films now incorporate a new version of radiotherapy film called Gafchromic EBT3 which utilizes a different physical design compared to EBT2. EBT3 has an equal 
125 micron thick polyester laminate of both sides of the sensitive layer. As such, we would expect EBT3 to possess different UV measurement properties and testing would need to be performed on this film type to ascertain its UV sensitivity. In terms of the effects of UV sensitivity of EBT2 on radiotherapy dosimetry, the higher UV response of the film through the thick laminate side means that storage and clinical use of EBT2 should be performed with the films thin layer facing upwards to minimize unwanted film darkening caused by ambient and UV light exposure.

EBT2 film has provided an adequate tool for the assessment and measurement of solar UV radiation. Some advantages of using such a film detector would include the ability to measure exposure over a two-dimensional area with a high degree of spatial resolution. The film is relatively inexpensive and easy to use. EBT2 film is also water proof so can be left outside for extended periods of time in rain or other environmental factors during measurements. Some disadvantages would be that it is less accurate than hand held meters and cannot measure an instantaneous UV irradiance. The film also must be placed in the correct orientation (up or down) for the entire experimental procedure for accurate results. However, with these considerations in mind, EBT2 film provides a new and novel tools for the measurement of solar UV radiation and will be advantageous for use in niche applications.

\section{Conclusion}

EBT2 Gafchromic film has been shown to be an adequate dosimeter for solar ultraviolet (UV) radiation when exposure is performed with the underside 'thick laminate' part of the film. Results have shown that accuracy and reproducibility of within $\pm 7 \%$ is achievable in this configuration. The EBT2 film, whilst having an UV radiation inhibitor within the film to minimize UV effects from the films top side thus allowing better handling performance for medical x-ray radiation dosimetry, still provides a tool for two-dimensional UV solar dosimetry for broad band UV measurements.

\section{Acknowledgments}

This work has been fully supported by grants from the State Key Laboratory (SKL) (project no. 9360136) and from the City University of Hong Kong (project no. City U 7008173).

\section{References}

Allen D J, Nogue S and Baker N R 1998 Ozone depletion and increased UV-B radiation: Is there a real threat to photosynthesis? J. Exp. Bot. 49 1775-88

Butson E, Alnawaf H, Yu P K N and Butson M J 2011 Scanner uniformity improvements for radiochromic film analysis with matt reflectance backing Australas. Phys. Eng. Sci. Med. 34 401-7

Butson E T, Cheung T, Yu P K N and Butson M J 2010 Measuring solar UV radiation with EBT radiochromic film Phys. Med. Biol. 55 N487-93

Butson M J, Cheung T, Yu P K N and Alnawaf H 2009 Dose and absorption spectra response of EBT2 Gafchromic film to high energy x-rays Australas. Phys. Eng. Sci. Med. 32 196-202

Butson M, Yu P and Metcalfe P 1998a Effects of readout light sources and ambient light on radiochromic film Phys. Med. Biol. 43 2407-12

Butson M J, Yu P and Metcalfe P 1998b Measurement of off-axis and peripheral skin dose using radiochromic film Phys. Med. Biol. 43 2647-50

Cheung J Y C, Yu K N, Yu C P and Ho R T K 1998 Monte Carlo calculation of single-beam dose profiles used in a gamma knife treatment planning system Med. Phys. 25 1673-5

Cheung T, Butson M J and Yu P K N 2006 Independence of calibration curves for EBT Gafchromic films of the size of high-energy x-ray fields Appl. Radiat. Isot. 64 1027-30 
Diffey B L 1982 Ultraviolet Radiation in Medicine (Bristol: Hilger)

ISO 2007 Space environment (natural and artificial)_process for determining solar irradiances ISO 21348 (Geneva: International Organization for Standardization)

ISP Corp 2009 Gafchromic EBT2, Self developing film for radiotherapy dosimetry ISP White Paper (Wayne, NJ: ISP Corporation)

Ohuchi H 2007 High sensitivity radiochromic film dosimetry using an optical common-mode rejection and a reflectivemode flatbed color scanner Med. Phys. 34 4207-12

Parisi A V and Kimlin M G 2004 Personal solar UV exposure measurements employing modified polysulphone with an extended dynamic range Photochem. Photobiol. 79 411-5

Schouten P W, Parisi A V and Turnbull D J 2007 Evaluation of a high exposure solar UV dosimeter for underwater use Photochem. Photobiol. 83 931-7

Schouten P W, Parisi A V and Turnbull D J 2009 Applicability of the polyphenylene oxide film dosimeter to high UV exposures in aquatic environments J. Photochem. Photobiol. B 96 184-92 